\title{
Myocardial involvement in eosinophilic granulomatosis with polyangiitis evaluated with cardiopulmonary magnetic resonance
}

\author{
Jakub Lagan ${ }^{1,2} \oplus$. Josephine H. Naish ${ }^{2}$. Christien Fortune ${ }^{1,2}$. Joshua Bradley ${ }^{1,2}$. David Clark ${ }^{1} \cdot$ Robert Niven $^{1,3}$. \\ Nazia Chaudhuri ${ }^{1,3} \cdot$ Erik B. Schelbert ${ }^{4,5,6} \cdot$ Matthias Schmitt $^{1,2} \cdot$ Christopher A. Miller $^{1,2,7}$
}

Received: 4 June 2020 / Accepted: 27 October 2020 / Published online: 19 November 2020

(C) The Author(s) 2020

\begin{abstract}
Patients with eosinophilic granulomatosis with polyangiitis (EGPA) most commonly die from cardiac causes, however, cardiac involvement remains poorly characterised and the relationship between cardiac and pulmonary disease is not known. This study aimed to characterise myocardial and pulmonary manifestations of EGPA, and their relationship. Prospective comprehensive cardiopulmonary investigation, including a novel combined cardiopulmonary magnetic resonance imaging (MRI) technology, was performed in 13 patients with stable EGPA. Comparison was made with 11 prospectively recruited matched healthy volunteers. Stable EGPA was associated with focal replacement and diffuse interstitial myocardial fibrosis (myocardial extracellular volume $26.9 \%$ vs. $24.7 \%$; $\mathrm{p}=0.034$ ), which drove a borderline increase in left ventricular mass $(56 \pm 9 \mathrm{~g} / \mathrm{m} 2$ vs. $49 \pm 8 \mathrm{~g} / \mathrm{m} 2 ; \mathrm{p}=0.065)$. Corrected QT interval was significantly prolonged and was associated with the severity of myocardial fibrosis $(\mathrm{r}=0.582, \mathrm{p}=0.037)$. Stable EGPA was not associated with increased myocardial capillary permeability or myocardial oedema. Pulmonary tissue perfusion and capillary permeability were normal and there was no evidence of pulmonary tissue oedema or fibrosis. Forced expiratory volume in one second showed a strong inverse relationship with myocardial fibrosis $(\mathrm{r}=-0.783, \mathrm{p}=0.038)$. In this exploratory study, stable EGPA was associated with focal replacement and diffuse interstitial myocardial fibrosis, but no evidence of myocardial or pulmonary inflammation or pulmonary fibrosis. Myocardial fibrosis was strongly associated with airway obstruction and abnormal cardiac repolarisation. Further investigation is required to determine the mechanisms underlying the association between heart and lung disease in EGPA and whether an immediate immunosuppressive strategy could prevent myocardial fibrosis formation.
\end{abstract}

Keywords Cardiac magnetic resonance scanning · Parametric mapping · Dynamic contrast enhanced magnetic resonance · Eosinophilic granulomatosis with polyangiitis $\cdot$ Churg $\cdot$ Strauss syndrome

Christopher A. Miller

Christopher.Miller@manchester.ac.uk

1 Manchester University NHS Foundation Trust, Wythenshawe Hospital, Southmoor Road, Wythenshawe, Manchester M23 9LT, UK

2 Division of Cardiovascular Sciences, School of Medical Sciences, Faculty of Biology, Medicine and Health, Manchester Academic Health Science Centre, University of Manchester, Oxford Road, Manchester M13 9PL, UK

3 Division of Infection, Immunity and Respiratory Medicine, School of Biological Sciences, Faculty of Biology, Medicine and Health, Manchester Academic Health Science Centre, University of Manchester, Oxford Road, Manchester M13 9PL, UK
4 Department of Medicine, University of Pittsburgh School of Medicine, Pittsburgh, PA, USA

5 UPMC Cardiovascular Magnetic Resonance Center, Heart and Vascular Institute, Pittsburgh, PA, USA

6 Clinical and Translational Science Institute, University of Pittsburgh, Pittsburgh, PA, USA

7 Division of Cell-Matrix Biology and Regenerative Medicine, School of Biology, Faculty of Biology, Medicine and Health, Manchester Academic Health Science Centre, Wellcome Centre for Cell-Matrix Research, University of Manchester, Oxford Road, Manchester M13 9PT, UK 


\section{Introduction}

Eosinophilic granulomatosis with polyangiitis (EGPA), formerly known as Churg-Strauss syndrome, is a hybrid condition comprising a systemic antineutrophil cytoplasmic antibody (ANCA)-associated vasculitis and a hypereosinophilic disorder with frequent lung involvement that is associated with asthma [1,2].

Whilst a wide range of cardiac manifestations are described, including pericarditis, pericardial effusion, tamponade, myocarditis, cardiomyopathy, myocardial infarction and heart failure [2], clinically evident cardiac manifestations are uncommon [3, 4]. In contrast, up to approximately $90 \%$ of patients have evidence of cardiac involvement at post-mortem and between a third and three quarters of patients with EGPA die from cardiac causes [4-7].

Magnetic resonance imaging (MRI) provides unparalleled evaluation of cardiac structure, function and tissue characterisation and its application in EGPA has provided insight into cardiac disease expression [5, 8-11]. However, contemporary cardiac MRI techniques, which provide quantitative characterisation of myocardial injury and adaptation, have been applied sparsely and the relationship between cardiac and pulmonary disease is not known.

In this study, we sought to apply a novel combined cardiopulmonary MRI technology, which provides comprehensive and simultaneous interrogation of cardiac and pulmonary structure, function and tissue character, in patients with stable EGPA in order to: (1) Characterise myocardial and pulmonary manifestations of EGPA; and (2) Investigate the relationship between cardiac and pulmonary disease in EGPA.

\section{Materials and methods}

\section{Study design}

This prospective research study aimed to apply a combined cardiopulmonary MRI protocol in patients with stable EGPA and matched healthy volunteers in order to investigate myocardial and pulmonary manifestations of EGPA, and their relationship.

\section{Study population}

Stable patients with a clinical diagnosis of EGPA according to the 2012 Chapel Hill consensus [1] were prospectively recruited at Manchester University NHS Foundation Trust. Age and gender matched volunteers with no cardiovascular symptoms, no history of any medical condition and normal electrocardiogram (ECG) were prospectively recruited as controls.

Exclusion criteria included: history of any cardiovascular disorder other than cardiac disease that was thought, by the attending clinical team, to be secondary to EGPA, contraindication to MRI, hypersensitivity to gadolinium-based contrast agent (GBCA), estimated glomerular filtration rate $<40 \mathrm{ml} / \mathrm{min} / 1.73 \mathrm{~m}^{2}$.

\section{Study procedures}

Participants underwent blood sampling for blood count, renal function, c-reactive protein, high sensitivity troponin $\mathrm{I}$, and B-human chorionic gonadotropin in females of childbearing potential, ECG and MRI scanning on the same day (see below). Clinical pulmonary function tests performed closest to the study visit were recorded.

\section{Combined cardiopulmonary MRI}

\section{Protocol}

MRI was performed at $1.5 \mathrm{~T}$ (Avanto, Siemens Medical Imaging). Scan duration $60 \mathrm{~min}$. It included the following sequences (Fig. 1):

1. Steady-state free precession (SSFP) cine imaging of the heart (standard long- and short-axis views) to provide measurement of cardiac function.

2. T1 mapping (by MOdified Look-Locker Inversion Recovery (MOLLI)) at basal and mid left ventricular (LV) short axis level before and 15 min following the

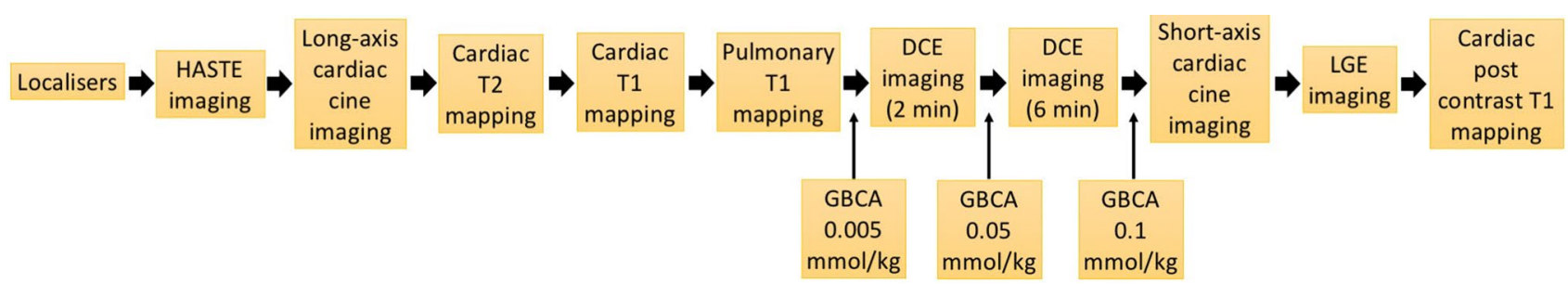

Fig. 1 Cardiopulmonary magnetic resonance imaging protocol. DCE dynamic contrast enhanced, GBCA gadolinium based contrast agent, HASTE half-fourier-acquired single-shot turbo spin echo, $L G E$ late enhancement imaging 
final bolus of GBCA (see below) to provide evaluation of myocardial oedema and fibrosis. T2 mapping (by T2-prepared SFFP) at the same locations to provide further assessment of myocardial oedema. Late gadolinium enhancement (LGE) imaging beginning at 6 min following the final GBCA bolus (see below) to assess for focal replacement myocardial fibrosis.

3. T1 mapping (by MOLLI) in the central sagittal plane of both lungs to provide evaluation of lung tissue injury.

4. Dynamic contrast enhanced (DCE) imaging using freebreathing 2D saturation-recovery fast low angle shot (FLASH) dynamic acquisitions in four planes (basal and mid LV short axis, left and right lung central sagittal planes) to assess myocardial and pulmonary capillary permeability (transfer constant $\left(\mathrm{K}^{\text {trans }}\right)$ ), pulmonary extracellular volume fraction $(\mathrm{Ve})$ and blood flow $(\mathrm{F})$. GBCA (gadoterate meglumine (Dotarem), Guerbet, France; $3 \mathrm{ml} / \mathrm{s}$ ) administration was divided into three doses in order to avoid saturation of the peak of the arterial input function (AIF), which occurs as a result of the non-linear relationship between contrast agent concentration and signal intensity, and T2 shortening effects, at higher contrast agent concentrations [12]. The initial low dose $(0.005 \mathrm{mmol} / \mathrm{kg})$ bolus was used to calculate the AIF first pass peak, followed by $2 \mathrm{~min}$ of dynamic acquisitions with a temporal resolution equal to $50 \%$ of heart rate. Then a higher GBCA dose $(0.05 \mathrm{mmol} / \mathrm{kg})$ was administered and followed by 6 min of dynamic acquisitions. The higher dose provided higher contrast to noise ratio for the tail of the AIF and for the myocardial and pulmonary tissue curves. The low dose curve was multiplied by 10 and combined with the high dose curve to produce the final AIFs for the kinetic analysis. The third contrast agent dose $(0.1 \mathrm{mmol} / \mathrm{kg})$ was administered after the dynamic acquisition to allow LGE imaging and post-contrast T1 mapping.

\section{Analysis}

Myocardial function Cardiac volumetric analysis was performed using Circle CVI42 (Circle Cardiovascular Imaging, Canada) according to current guidelines [13].

Myocardial and lung tissue characterisation T1 and T2 maps were generated in Siemens Argus (Siemens Medical Imaging) and transferred into Horos (Horos $2 \mathrm{~K}$ v2.2.0 The Horos Project) where epicardial, endocardial and blood pool regions of interest (ROI) were drawn. Partial volume effects of blood were minimised in myocardial ROIs by using the middle third of myocardium. Lung borders were contoured in Horos, excluding the cardiac outline, to define wholelung ROI. Individual lobes were identified as previously described [14]. Myocardial extracellular volume (ECV) was calculated using same-day haematocrit as described previously [15]. LV extracellular matrix mass (g) was calculated by multiplying LV mass by ECV [16]. LV cellular mass (g) was calculated by multiplying LV mass by $(100 \%-\mathrm{ECV})$.

Myocardial and lung blood flow characteristics DCE imaging was analysed using custom written Matlab code (v9.0, The MathWorks, USA). Cardiac motion correction was achieved using an intensity-based rigid registration algorithm. Lung registration was performed using Advanced Normalisation Tools (ANTs) symmetric normalization non-linear registration algorithm, employing cross-correlation as the similarity measure [17]. Epicardial, endocardial and lung ROIs were drawn as described above. AIFs were derived from the right ventricular (RV) blood pool for the lungs and the LV blood pool for the myocardium. Care was taken to avoid any trabeculations or papillary muscles. Contrast agent kinetics were modelled using an extended version of a Kety model on a voxel-by-voxel basis within registered ROIs to calculate $\mathrm{K}^{\text {trans }}$ and Ve [18]. Pulmonary blood flow (F) was calculated by deconvolution of the first pass dynamic data as described previously $[18,19]$.

\section{Statistical analysis}

This was an exploratory study, and there were limited data upon which to base a power calculation. As a guide, 10 patients were required in each group to detect an absolute minimum difference, between EGPA and healthy control groups, in myocardial ECV of 3\% [10], with $80 \%$ power at a 5\% significance level (2-sided), assuming a standard deviation of $2.2 \%$ within each group [10,11, 15]. Data distribution was determined using the Shapiro-Wilk test. Data are summarised using mean and standard deviation or median and interquartile range (IQR), and were compared using t tests or non-parametric equivalents, as appropriate. Analyses were performed using SPSS (version 22, IBM, USA).

\section{Results}

\section{Participant characteristics}

Thirteen patients with stable EGPA and 11 healthy volunteers were recruited (Table 1). Groups were matched for age and gender. Twelve (92\%) patients with EGPA were receiving immunosuppressive therapy at the time of the study. Patients with EGPA had a significantly higher white cell count ( 10.7 vs. $\left.6.0 \times 10^{9} / \mathrm{L} ; \mathrm{p}=0.001\right)$, however, eosinophil count was no different $\left(0.23\right.$ vs. $\left.0.11 \times 10^{9} / L ; p=0.23\right)$. High sensitivity troponin I was within the normal range in all participants. Pulmonary function tests were available in seven patients with EGPA. 
Table 1 Participant characteristics

\begin{tabular}{|c|c|c|c|}
\hline Parameter & Controls $(n=11)$ & EGPA $(n=13)$ & $\mathrm{p}$ value \\
\hline \multicolumn{4}{|l|}{ Demographics } \\
\hline Age (years) & $53(46-56)$ & $54(49-57)$ & 0.543 \\
\hline Gender (female, \%) & $4(36 \%)$ & $6(46 \%)$ & 0.697 \\
\hline $\operatorname{BSA}\left(\mathrm{m}^{2}\right)$ & $1.98(1.73-2.21)$ & $2.22(1.8-2.3)$ & 0.369 \\
\hline \multicolumn{4}{|l|}{ EGPA manifestations } \\
\hline ANCA positive & $0(0 \%)$ & $3(23 \%)$ & \\
\hline Asthma & $0(0 \%)$ & $12(92 \%)$ & \\
\hline History of eosinophilia & $0(0 \%)$ & $13(100 \%)$ & \\
\hline Peripheral neuropathy & $0(0 \%)$ & $10(77 \%)$ & \\
\hline Paranasal sinus abnormalities & $0(0 \%)$ & $6(46 \%)$ & \\
\hline Extravascular eosinophils & $0(0 \%)$ & $2(15 \%)$ & \\
\hline Cardiac involvement & $0(0 \%)$ & $2(15 \%)$ & \\
\hline Renal involvement & $0(0 \%)$ & $2(15 \%)$ & \\
\hline GI involvement & $0(0 \%)$ & $2(15 \%)$ & \\
\hline Dermatological involvement & $0(0 \%)$ & $2(15 \%)$ & \\
\hline Polymyalgia & $0(0 \%)$ & $2(15 \%)$ & \\
\hline Hypertension & $0(0 \%)$ & $2(15 \%)$ & \\
\hline Alveolar haemorrhage & $0(0 \%)$ & $1(8 \%)$ & \\
\hline \multicolumn{4}{|l|}{ Medications } \\
\hline Oral steroids & $0(0 \%)$ & $12(92 \%)$ & \\
\hline Mycophenolate mofetil & $0(0 \%)$ & $6(46 \%)$ & \\
\hline Methotrexate & $0(0 \%)$ & $2(15 \%)$ & \\
\hline Inhaled steroids & $0(0 \%)$ & $11(85 \%)$ & \\
\hline Inhaled LABAs & $0(0 \%)$ & $9(69 \%)$ & \\
\hline Inhaled SABAs & $0(0 \%)$ & $12(92 \%)$ & \\
\hline Inhaled LAMAs & $0(0 \%)$ & $4(31 \%)$ & \\
\hline Theophylline & $0(0 \%)$ & $2(15 \%)$ & \\
\hline LTRA & $0(0 \%)$ & $2(15 \%)$ & \\
\hline Macrolides & $0(0 \%)$ & $3(23 \%)$ & \\
\hline Statins & $0(0 \%)$ & $4(31 \%)$ & \\
\hline ACEI/sartans & $0(0 \%)$ & $3(23 \%)$ & \\
\hline B Blockers & $0(0 \%)$ & $1(8 \%)$ & \\
\hline Calcium channel blockers & $0(0 \%)$ & $1(8 \%)$ & \\
\hline Duretics & $0(0 \%)$ & $3(23 \%)$ & \\
\hline Antiplatelets & $0(0 \%)$ & $1(8 \%)$ & \\
\hline Anticoagulants & $0(0 \%)$ & $2(15 \%)$ & \\
\hline \multicolumn{4}{|l|}{ Lung function tests } \\
\hline $\mathrm{FEV}_{1}(\mathrm{~L} / \mathrm{s})$ & & $2.4 \pm 0.7^{*}$ & \\
\hline $\mathrm{FEV}_{1} \%(\%)$ & & $70.6 \pm 25.0^{*}$ & \\
\hline FVC (L) & & $3.6 \pm 1.0^{*}$ & \\
\hline FVC $\%(\%)$ & & $85.0 \pm 18.8^{*}$ & \\
\hline $\mathrm{FEV}_{1} / \mathrm{FVC}$ & & $0.68 \pm 0.13^{*}$ & \\
\hline \multicolumn{4}{|l|}{ Laboratory findings } \\
\hline $\mathrm{CRP}(\mathrm{mg} / \mathrm{L})$ & $1(0-2)$ & $3(1-10)$ & 0.070 \\
\hline $\mathrm{WBC}\left(\times 10^{9} / \mathrm{L}\right)$ & $6(4.9-7.1)$ & $10.7(9.8-12.85)$ & 0.001 \\
\hline Eosinophil count $\left(\times 10^{9} / \mathrm{L}\right)$ & $0.11(0.07-0.21)$ & $0.23(0.07-0.44)$ & 0.213 \\
\hline Troponin I (ng/L) & $4(4-4)$ & $4(4-4)$ & 0.317 \\
\hline \multicolumn{4}{|l|}{ ECG findings } \\
\hline PR duration (ms) & $160(130-160)$ & $160(140-160)$ & 0.975 \\
\hline QRS duration (ms) & $80(80-100)$ & $80(70-80)$ & 0.115 \\
\hline QTc (ms) & $398 \pm 18$ & $414 \pm 17$ & 0.043 \\
\hline
\end{tabular}


Table 1 (continued)

\begin{tabular}{llll}
\hline Parameter & Controls $(\mathrm{n}=11)$ & EGPA $(\mathrm{n}=13)$ & $\mathrm{p}$ value \\
\hline ST-T abnormalities (present) & $0(0 \%)$ & $4(31 \%)$ & 0.098 \\
\hline
\end{tabular}

Data presented as mean \pm standard deviation or median (interquartile range) depending on data distribution $A C E I$ angiotensin converting enzyme inhibitor, $A N C A$ antineutrophil cytoplasmic antibodies, $B S A$ body surface area, $C R P$ c reactive protein, $E G P A$ eosinophilic granulomatosis with polyangiitis, $F E V_{l}$ forced expiratory volume in one second, $F E V_{l} \%$ percentage of predicted forced expiratory volume in one second, $F V C$ forced vital capacity, $F V C \%$ percentage of predicted forced vital capacity GI-gastrointestinal tract, $L A B A$ long acting B agonist, LAMA long acting muscarinic antagonist, LTRA leukotriene receptor antagonist, $L V H$ left ventricular hypertrophy, $S A B A$ short acting B agonist, $W B C$ white blood cell

${ }^{*} \mathrm{~N}=7$

Two patients with EGPA had a history of cardiac involvement; one patient had previously had a pericardial effusion and a pericardial window formed, one patient had experienced a distal coronary artery dissection at a young age.

Corrected QT interval was significantly longer in EGPA than in healthy volunteers $(414 \pm 17$ vs. $398 \pm 18 \mathrm{~ms}$; $\mathrm{p}=0.04)$. Four patients with EGPA exhibited ST segment (ST elevation in one; ST depression in one) or T wave abnormalities ( $\mathrm{T}$ wave inversion in two). No patients exhibited clinically significant arrhythmias.

\section{Cardiopulmonary manifestations}

Ventricular size, ventricular ejection fraction and atrial size were no different in EGPA compared to control (Table 2). Body surface area-indexed LV mass was borderline higher in EGPA ( $56 \pm 9$ vs. $49 \pm 8 \mathrm{~g} / \mathrm{m} 2 ; \mathrm{p}=0.065)$.

EGPA was associated with elevated myocardial ECV (26.9\% (25.9-28.5\%) vs $24.7 \%$ (23.6-26.4\%); $\mathrm{p}=0.034)$, increased extracellular matrix mass ( $24 \pm 6$ vs. $32 \pm 9 ; \mathrm{p}=0.015)$ and elevated native T1 $(1063 \pm 46$ vs. $1003 \pm 21 \mathrm{~ms} ; \mathrm{p}=0.001)$. Ten patients with EGPA exhibited non-ischaemic LGE (mid myocardial wall in five, epicardial in one, RV insertion point in nine; $\mathrm{p}<0.001$; (some patients displayed more than one type)). Myocardial T2 and capillary permeability were no different in EGPA compared to control. Figure 2 illustrates representative examples of tissue characterisation in a patient with EGPA.

There was no relationship between corrected QT interval and medications. Corrected QT interval was significantly associated with myocardial ECV interval $(r=0.582$, $\mathrm{p}=0.037$; Fig. 3a).

Two patients had evidence of a myocardial infarction (MI) on LGE imaging: in one patient it was previously unrecognised and the other was the patient with a history of coronary artery dissection.

Pulmonary tissue T1, capillary permeability, extracellular volume fraction and blood flow were no different in EGPA compared to healthy volunteers (Table 3).

\section{Relationship between cardiac and pulmonary manifestations}

Forced expiratory volume in one second $\left(\mathrm{FEV}_{1}\right)$ was strongly negatively correlated to native myocardial $\mathrm{T} 1(\mathrm{r}=-0.816$, $\mathrm{p}=0.025)$ and ECV $(r=-0.783, p=0.038$; Fig. $3 b)$. There were no significant correlations between myocardial and pulmonary MRI measurements, and no association between circulating eosinophil levels and cardiac findings.

\section{Discussion}

In the first study to utilise a combined multiparametric cardiopulmonary MRI protocol in EGPA, we demonstrate a number of novel findings. We show that stable EGPA is associated with focal replacement and diffuse interstitial myocardial fibrosis, which drives a borderline increase in LV mass, but is not associated with myocardial oedema or increased capillary permeability. We show that myocardial fibrosis burden is associated with abnormal cardiac repolarisation. We demonstrate that in the setting of stable disease, EGPA is associated with normal pulmonary tissue perfusion and no evidence of pulmonary inflammation or fibrosis. Finally, we show that myocardial fibrosis burden is strongly related to the severity of airway obstruction.

It is increasingly recognised that conditions considered to be primary pulmonary disorders are associated with cardiovascular conditions, particularly heart failure, independent of shared risk factors, and that in turn, cardiovascular conditions independently contribute to all-cause mortality in patients with pulmonary conditions [20].

EGPA has dual disease categorisation, being classified within the small to medium vessel vasculitides associated with ANCA and in the hypereosinophilic syndromes [2]. It is characterised histologically by the presence of a small to medium vessel necrotising vasculitis and eosinophil-rich, necrotising granuloma, which often involve the respiratory tract. Both vessel inflammation and eosinophilic proliferation are thought to contribute to organ damage [1]. 
Table 2 Myocardial magnetic resonance imaging indices

\begin{tabular}{|c|c|c|c|}
\hline Parameter & Controls $(n=11)$ & EGPA $(n=13)$ & $\mathrm{p}$ value \\
\hline \multicolumn{4}{|l|}{ LV } \\
\hline LV EDV/BSA $\left(\mathrm{ml} / \mathrm{m}^{2}\right)$ & $76 \pm 11$ & $76 \pm 18$ & 0.932 \\
\hline LV ESV/BSA $\left(\mathrm{ml} / \mathrm{m}^{2}\right)$ & $30 \pm 6$ & $31 \pm 14$ & 0.770 \\
\hline LV EF (\%) & $62 \pm 5$ & $60 \pm 9$ & 0.645 \\
\hline LV mass/BSA $\left(\mathrm{g} / \mathrm{m}^{2}\right)$ & $49 \pm 8$ & $56 \pm 9$ & 0.065 \\
\hline \multicolumn{4}{|l|}{ RV } \\
\hline RV EDV/BSA $\left(\mathrm{ml} / \mathrm{m}^{2}\right)$ & $83 \pm 14$ & $77 \pm 16$ & 0.305 \\
\hline RV ESV/BSA (ml/m²) & $37 \pm 7$ & $33 \pm 10$ & 0.396 \\
\hline RV EF (\%) & $56 \pm 4$ & $56 \pm 8$ & 0.996 \\
\hline \multicolumn{4}{|l|}{ Pericardium } \\
\hline Pericardial effusion (n,\%) & $0(0 \%)$ & $1(8 \%)$ & 1.000 \\
\hline Pericardial thickening $(\mathrm{n}, \%)$ & $0(0 \%)$ & $2(15 \%)$ & 0.482 \\
\hline \multicolumn{4}{|l|}{ Atria } \\
\hline $\mathrm{LA}$ area/BSA $\left(\mathrm{cm}^{2} / \mathrm{m}^{2}\right)$ & $12 \pm 1$ & $12 \pm 3$ & 0.319 \\
\hline $\mathrm{RA}$ area/BSA $\left(\mathrm{cm}^{2} / \mathrm{m}^{2}\right)$ & $12 \pm 3$ & $11 \pm 2$ & 0.221 \\
\hline \multicolumn{4}{|l|}{ Tissue characterisation } \\
\hline Ischaemic LGE (present) & $0(0 \%)$ & $2(15 \%)$ & 0.482 \\
\hline Ischaemic LGE (g) & $0.00(0.00-0.00)$ & $0.00(0.00-0.00)$ & 0.184 \\
\hline Non-ischaemic LGE (present) & $0(0 \%)$ & $10(77 \%)$ & $<0.001$ \\
\hline Non-ischaemic LGE (g) & $0.00(0.00-0.00)$ & $0.51(0.12-3.16)$ & 0.001 \\
\hline $\mathrm{T} 2(\mathrm{~ms})$ & $50 \pm 2$ & $50 \pm 3$ & 0.533 \\
\hline $\mathrm{T} 1$ (ms) & $1003 \pm 21$ & $1063 \pm 46$ & 0.001 \\
\hline $\operatorname{ECV}(\%)$ & $24.7(23.6-26.4)$ & $26.9(25.9-28.5)$ & 0.034 \\
\hline Extracellular matrix mass (g) & $24 \pm 6$ & $32 \pm 9$ & 0.015 \\
\hline Cellular mass $(\mathrm{g})$ & $74 \pm 21$ & $84 \pm 16$ & 0.191 \\
\hline Median $\mathrm{K}^{\text {trans }}\left(\min ^{-1}\right)$ & $0.32 \pm 0.08$ & $0.31 \pm 0.08$ & 0.762 \\
\hline
\end{tabular}

Data presented as mean \pm standard deviation or median (interquartile range) depending on data distribution $E C V$ extracellular volume fraction, $E D V$ end systolic volume, $E F$ ejection fraction, $E S V$ end systolic volume, $K^{\text {trans }}$ transfer constant, $L A$ left atrium, $L G E$ late gadolinium enhancement, $L V$ left ventricle, $R A$ right atrium, $R V$ right ventricle, $S V$ stroke volume. Other abbreviations as per Table 1

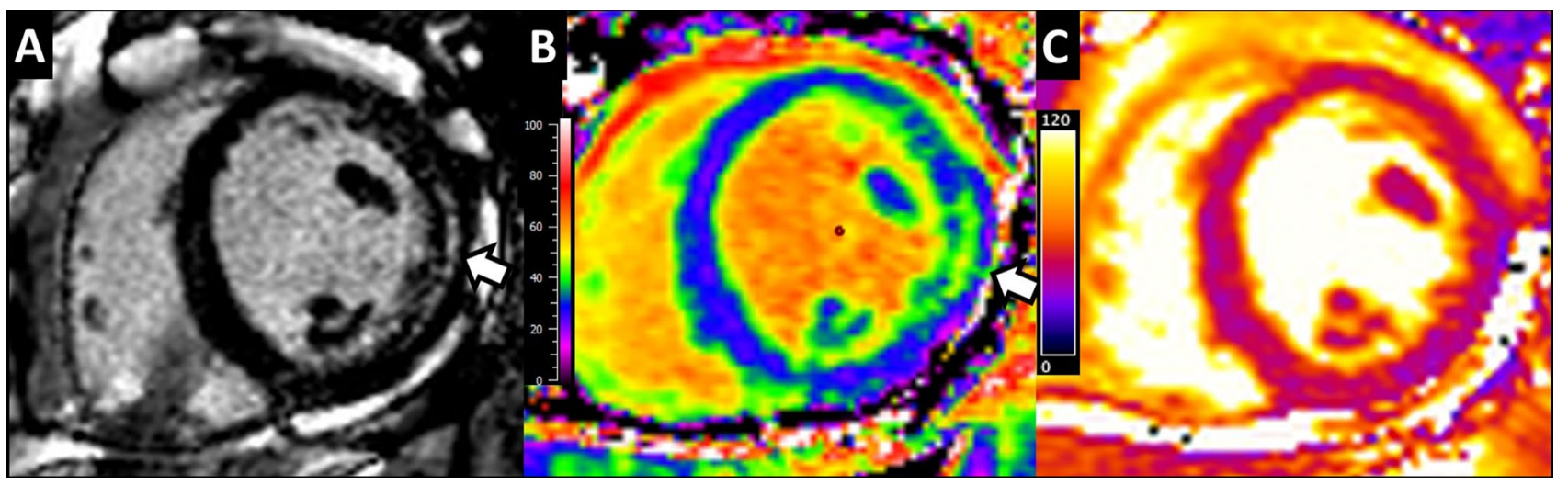

Fig. 2 Myocardial tissue characterisation in a patient with eosinophilic granulomatosis with polyangiitis. a On late enhancement imaging (arrow) and $\mathbf{b}$ extracellular volume mapping (extracellular volume in the lateral wall is elevated at $37 \%$ ), there is evidence of non-ischae- mic fibrosis in the lateral wall of the left ventricle. c On T2 mapping, there is no evidence of myocardial inflammation (lateral wall: $45 \mathrm{~ms}$; septum: $47 \mathrm{~ms}$ ) 


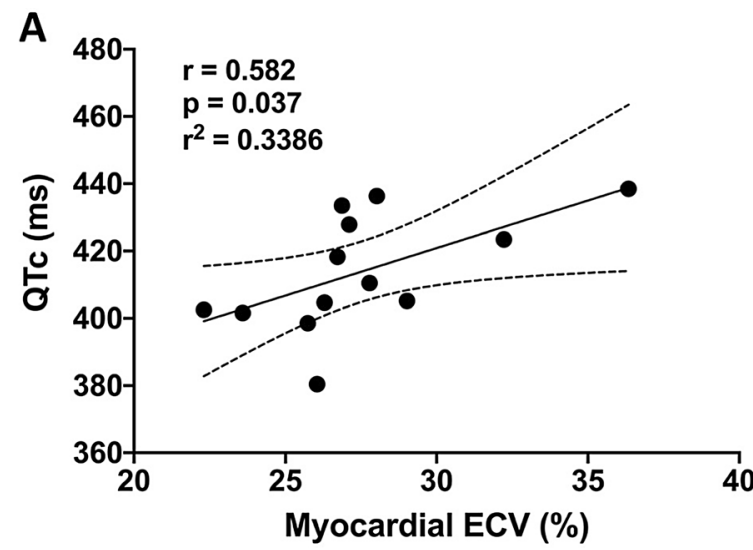

Fig. 3 Relationship between myocardial extracellular volume (ECV) fraction, lung function and cardiac repolarisation in eosinophilic granulomatosis with polyangiitis. a Corrected QT interval was significantly associated with myocardial fibrosis burden, measured using

Table 3 Pulmonary magnetic resonance imaging indices

\begin{tabular}{llll}
\hline Parameter & Controls $(\mathrm{n}=11)$ & EGPA $(\mathrm{n}=13)$ & $\mathrm{p}$ value \\
\hline $\mathrm{T} 1(\mathrm{~ms})$ & $1261 \pm 46$ & $1290 \pm 65$ & 0.232 \\
Median Ve (\%) & $\begin{array}{c}20.8(18.73- \\
26.86)\end{array}$ & $\begin{array}{c}21.9(18.62- \\
25.64)\end{array}$ & 0.839 \\
& $0.25 \pm 0.07$ & $0.23 \pm 0.09$ & 0.561 \\
$\begin{array}{l}\text { Median K } \\
\left(\mathrm{min}^{-1}\right)\end{array}$ & $2.32(1.76-3.10)$ & $2.77(1.88-3.15)$ & 0.794 \\
$\begin{array}{l}\text { Median F }(\mathrm{ml} \\
\text { blood/ml tissue/ } \\
\text { min })\end{array}$ & & & \\
\hline
\end{tabular}

Data presented as mean \pm standard deviation or median (interquartile range) depending on data distribution

$F$ Pulmonary tissue blood flow, Ve pulmonary extracellular volume fraction. Other abbreviations as per Table 2

The cardiac pathogenesis is that of eosinophilic myocardial infiltration that rapidly progresses to myocardial fibrosis, which chronically leads to dilated or restrictive cardiomyopathy [2]. Cardiac involvement often occurs early in the disease process [8]. Some studies report that cardiac involvement is more common in ANCA-negative patients and is related to the degree of blood eosinophilia, but others have not found these associations $[4,8,21]$. Cardiomyopathy is independently associated with early death in patients with EGPA, and cardiac involvement potentially accounts for up to $80 \%$ of deaths $[2,3,7]$.

Patients all had stable EGPA, as evidenced by low levels of circulating $\mathrm{C}$ reactive protein and eosinophil counts (the slightly elevated white cell count is likely because $92 \%$ of patients were receiving corticosteroids). Perhaps as a result, we did not find an association between cardiac manifestations and circulating eosinophil levels. Seventy-seven percent of patients were ANCA-negative at diagnosis.

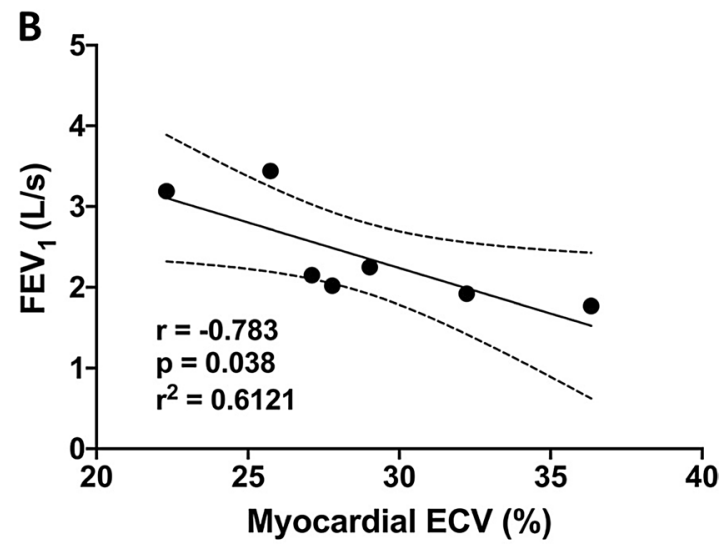

extracellular volume fraction (ECV). b Forced expiratory volume in one second $\left(\mathrm{FEV}_{1}\right)$ was significantly negatively correlated to myocardial fibrosis burden, measured using ECV

The multiparametric nature of MRI, i.e. its ability to measure multiple aspects of tissue structure, function, injury and adaptation within one scan, without radiation, means that it is uniquely placed to elucidate underlying disease mechanisms and understand their relationships. Myocardial ECV, measured using cardiac MRI, quantifies myocardial extracellular matrix volume and in the absence of significant inflammation, is a robust measure of myocardial fibrosis, having been extensively validated against histological collagen volume fraction [16, 22, 23]. Myocardial T1 is a surrogate of ECV, having also been validated against histological collagen. Myocardial LGE provides robust identification of focal replacement fibrosis [24, 25]. Myocardial T2 is directly proportional to myocardial water content [26] and $\mathrm{K}^{\text {trans }}$ measures capillary permeability [27]; both are applied as measures of tissue inflammation.

Using these techniques, we show that stable EGPA is associated with myocardial fibrosis (elevated ECV and T1) and an absence of myocardial inflammation (normal $\mathrm{T} 2$ and $\mathrm{K}^{\text {trans }}$ ), findings which are in keeping with the known histology, described earlier. Our findings also show that the evident myocardial fibrosis comprises both diffuse interstitial and replacement components, and that it is myocardial fibrosis that drives the borderline left ventricular hypertrophy i.e. rather than cellular hypertrophy.

Greulich et al. reported elevated myocardial ECV and T1 in EGPA, although myocardial T2 was also elevated, indicating the presence of myocardial oedema [10]. ECV and $\mathrm{T} 1 \mathrm{can}$ be affected by oedema, thus it is unclear whether the elevated ECV and T1 that Greulich et al. report represented myocardial oedema or fibrosis. In a study by Cereda et al., elevated ECV was confined to patients with reduced LV ejection fraction [11]. The prevalence of non-ischaemic focal replacement fibrosis in our study (77\%) is in keeping 
with that in previous studies [11,28-30]. Although quantitative T2 mapping has only been applied in one previous study in EPGA, studies using semi-quantitative T2-weighted imaging, and positron emission tomography, have generally shown a low prevalence of inflammation in stable EGPA, which is in keeping with the described histology and our findings [5, 9, 29-31].

Myocardial fibrosis is strongly associated with adverse outcome across a range of cardiovascular conditions [32, 33] and further work is required to determine the association between myocardial fibrosis and outcome in EGPA. Arrhythmia is a common feature of EGPA and it is of note that we demonstrate an association between myocardial fibrosis and corrected QT interval.

The authors of pathological studies demonstrating that myocardial fibrosis develops rapidly during the course of EGPA have suggested immediate and aggressive immunosuppressant treatment may be beneficial in preventing the development of heart failure and other cardiac complications $[2,8]$. Future studies applying MRI in the initial stages of EGPA are required in order to characterise the early myocardial manifestations and evaluate the impact of such an immunosuppressant strategy.

Importantly, one patient (8\%) was found to have an otherwise unrecognised MI. MI that is not detectable from patient history or ECG is well described in non-EGPA populations. Cardiac MRI with LGE has a sensitivity such that it is able to detect $0.7 \mathrm{~g}$ of infarcted myocardium [34]. Several large community cohort studies (including people with or at risk of cardiovascular disease) utilising cardiac MRI have demonstrated a significant prevalence of otherwise unrecognised MI, and a high associated mortality risk [35]. Subendocardial LGE is well described in EGPA but studies rarely comment on its apparent aetiology (i.e. non-ischaemic eosinophilic subendocardial fibrosis vs. ischaemic), or its context (i.e. history of MI). It is well recognized that EGPA is associated with coronary artery disease, and while our study is small, it suggests that unrecognised MI may not be uncommon. Given that unrecognised MI is only detectable using cardiac MRI, and that its detection allows initiation of secondary preventative therapy, consideration should be given to performing cardiac MRI in all patients with EGPA.

Demonstrating the unique utility of cardiac MRI, the cardiac MRI findings were not evident from circulating biomarkers (e.g. high sensitivity troponin) or on ECG. Furthermore, despite there being evidence of significant myocardial injury and adaptation, ventricular size and ejection fraction were normal, which is in keeping with most previous studies in EGPA, demonstrating the low sensitivity of ventricular size and ejection fraction for detecting cardiac injury [10, $28,30]$.

Our cardiopulmonary MRI protocol, which simultaneously interrogates cardiac and pulmonary inflammation, fibrosis and blood flow characteristics, provides a methodology to directly investigate cardiac and pulmonary pathophysiology, and their relationship. In the first study to apply pulmonary MRI in EGPA, we show that that in the setting of stable EGPA with effective immunosuppression, pulmonary tissue characterisation is normal with no evidence of inflammation or pulmonary fibrosis. Active pulmonary inflammation is associated with increased pulmonary T1, capillary permeability and pulmonary tissue blood flow [27, $36,37]$. Our findings, which showed no elevation in these markers, indicates stable EGPA is not associated with active pulmonary inflammation, which was in keeping with the clinical picture. Pulmonary T1 is reduced in the context of pulmonary tissue destruction, such as occurs with emphysema, and with pulmonary fibrosis [36, 38-42]. Histological studies have demonstrated that stable EGPA is not associated with emphysema or fibrosis [2, 6, 43, 44], which is in keeping with our findings that showed no reduction in pulmonary $\mathrm{T} 1$ and no increase in extracellular volume fraction in EGPA compared to controls. Indeed, a benefit of our combined cardio-pulmonary protocol is that it enabled us to demonstrate that myocardial fibrosis occurs independently of pulmonary fibrosis in EGPA.

We found a strong association between myocardial fibrosis and the severity of airflow limitation. This is a novel finding and the underlying mechanism is unclear. Associations between $\mathrm{FEV}_{1}$ and incident heart failure, and between $\mathrm{FEV}_{1}$ and adverse outcome in patients with $\mathrm{HF}$, particularly heart failure with preserved ejection fraction, are widely recognised [45-49]. The mechanisms underlying these associations are also not known, but, in the context of chronic obstructive pulmonary disease (COPD), circulating inflammatory markers are associated with incident heart failure, myocardial injury (circulating troponin level) and airflow obstruction [50-52]. In COPD, it has been hypothesised that systemic inflammation leading to myocardial injury and subsequent myocardial fibrosis may be the unifying mechanism [53]. It is possible that a similar mechanism may be responsible for the relationship between myocardial fibrosis and airflow limitation that we have demonstrated in EGPA, indeed our findings may shed light on these wider pulmonary-cardiac associations, but further work is required to investigate these mechanisms.

\section{Limitations}

The number of patients included was small, although we were able to demonstrate significant results despite the small numbers. EGPA is a rare condition with estimated prevalence of 2 to $22 \mathrm{per} / \mathrm{million}$ and the size of our study is in keeping with other recent studies [2, 10, 11]. It was not possible to obtain histological data; it was not felt ethically 
acceptable to conduct lung and endomyocardial biopsies as part of this study, and these procedures are not generally performed in stable EGPA patients.

\section{Conclusions}

In this exploratory study that utilised a combined multiparametric cardiopulmonary MRI protocol, we demonstrate that stable EGPA is associated with focal replacement and diffuse interstitial myocardial fibrosis, but no evidence of myocardial or pulmonary inflammation or pulmonary fibrosis. Myocardial fibrosis is strongly related to airway obstruction and abnormal cardiac repolarisation. Further investigation is required to determine the mechanisms underlying the association between heart and lung disease in EGPA, the impact myocardial fibrosis has on prognosis, and whether an immediate immunosuppressive strategy could prevent myocardial fibrosis formation.

Acknowledgements The authors would like to express their gratitude to Siemens Healthcare Ltd for technical support.

Author contributions JL - study conceptualisation and design, ethical approval application, recruitment, MRI acquisition and analysis, clinical data analysis, statistical analysis, writing and revision of the manuscript. JHN - MRI protocol and analysis tools design, revision of the manuscript. CF - recruitment and clinical data analysis, revision of the manuscript. JB - MRI analysis, revision of the manuscript. DC - MRI acquisition and analysis, revision of the manuscript. RN, $\mathrm{NC}$ - study conceptualisation and design, patient recruitment, revision of the manuscript. EBS, MS - study conceptualisation and design, revision of the manuscript. CAM - chief investigator for the study, study conceptualisation and design, supervision of all study aspects, writing and revision of the manuscript.

Funding Dr Lagan is funded by a Clinical Research Training Fellowship from the British Heart Foundation (FS/17/47/32805). Dr Miller is funded by a Clinician Scientist Award (CS-2015-15-003) from the National Institute for Health Research. The work was also supported in part by a British Heart Foundation Accelerator award to The University of Manchester (AA/18/4/34221). The views expressed in this publication are those of the authors and not necessarily those of the NHS, the National Institute for Health Research or the Department of Health. None of these funding bodies had a role in study design, data collection, analysis or interpretation or writing of the manuscript.

Data availability The datasets generated and/or analysed during the current study are available from the corresponding author on reasonable request.

\section{Compliance with ethical standards}

Conflict of interest Dr Miller received research support provided by Roche and Guerbert. Dr Miller served on an advisory board for Novartis and serves as an advisor for HAYA therapeutics. Other authors declare that they have no competing interests. Authors have full control of all primary data and agree to allow the journal to review the data if requested.
Ethics approval and consent to participate A North West - Greater Manchester West Research Ethics Committee of the UK National Research Ethics Service approved the study and written informed consent was obtained from all participants. The work was conducted according to the Helsinki Declaration.

Open Access This article is licensed under a Creative Commons Attribution 4.0 International License, which permits use, sharing, adaptation, distribution and reproduction in any medium or format, as long as you give appropriate credit to the original author(s) and the source, provide a link to the Creative Commons licence, and indicate if changes were made. The images or other third party material in this article are included in the article's Creative Commons licence, unless indicated otherwise in a credit line to the material. If material is not included in the article's Creative Commons licence and your intended use is not permitted by statutory regulation or exceeds the permitted use, you will need to obtain permission directly from the copyright holder. To view a copy of this licence, visit http://creativecommons.org/licenses/by/4.0/.

\section{References}

1. Jennette JC, Falk RJ, Bacon PA, Basu N, Cid MC, Ferrario F, et al. 2012 revised International Chapel Hill Consensus Conference Nomenclature of Vasculitides. Arthritis Rheum; Jan2013. p. $1-11$.

2. Mahr A, Moosig F, Neumann T, Szczeklik W, Taillé C, Vaglio A et al (2014) Eosinophilic granulomatosis with polyangiitis (churgStrauss): evolutions in classification, etiopathogenesis, assessment and management. Curr Opin Rheumatol 26(1):16-23

3. Guillevin L, Cohen P, Gayraud M, Lhote F, Jarrousse B, Casassus $P$ (1999) Churg-strauss syndrome. Clinical study and long-term follow-up of 96 patients. Medicine 78(1):26-37

4. Comarmond C, Pagnoux C, Khellaf M, Cordier J-F, Hamidou M, Viallard J-F et al (2013) Eosinophilic granulomatosis with polyangiitis (Churg-Strauss): clinical characteristics and long-term followup of the 383 patients enrolled in the French vasculitis study group cohort. Arthritis Rheum 65(1):270-281

5. Dennert RM, van Paassen P, Schalla S, Kuznetsova T, Alzand BS, Staessen JA et al (2010) Cardiac involvement in churg-Strauss syndrome. Arthritis Rheum 62(2):627-634

6. Churg J, Strauss L (1951) Allergic granulomatosis, allergic angiitis, and periarteritis nodosa. Am J Pathol 27(2):277-301

7. Bourgarit A, Le Toumelin P, Pagnoux C, Cohen P, Mahr A, Le Guern V et al (2005) Deaths occurring during the first year after treatment onset for polyarteritis nodosa, microscopic polyangiitis, and churg-Strauss syndrome: a retrospective analysis of causes and factors predictive of mortality based on 595 patients. Medicine (Baltimore) 84(5):323-330

8. Neumann T, Manger B, Schmid M, Kroegel C, Hansch A, Kaiser WA et al (2009) Cardiac involvement in churg-strauss syndrome: impact of endomyocarditis. Medicine (Baltimore) 88(4):236-243

9. Hazebroek MR, Kemna MJ, Schalla S, Sanders-van Wijk S, Gerretsen SC, Dennert R et al (2015) Prevalence and prognostic relevance of cardiac involvement in ANCA-associated vasculitis: eosinophilic granulomatosis with polyangiitis and granulomatosis with polyangiitis. Int J Cardiol 199:170-179

10. Greulich S, Mayr A, Kitterer D, Latus J, Henes J, Steubing H et al (2017) T1 and T2 mapping for evaluation of myocardial involvement in patients with ANCA-associated vasculitides. J Cardiovasc Magn Reson 19(1):6

11. Cereda AF, Pedrotti P, De Capitani L, Giannattasio C, Roghi A (2017) Comprehensive evaluation of cardiac involvement in 
eosinophilic granulomatosis with polyangiitis (EGPA) with cardiac magnetic resonance. Eur J Intern Med 39:51-56

12. Roberts TP (1997) Physiologic measurements by contrastenhanced MR imaging: expectations and limitations. J Magn Reson Imaging 7(1):82-90

13. Schulz-Menger J, Bluemke DA, Bremerich J, Flamm SD, Fogel MA, Friedrich MG et al (2013) Standardized image interpretation and post processing in cardiovascular magnetic resonance: society for cardiovascular magnetic resonance (SCMR) board of trustees task force on standardized post processing. J Cardiovasc Magn Reson 15(1):35

14. Donnola SB, Dasenbrook EC, Weaver D, Lu L, Gupta K, Prabhakaran A et al (2017) Preliminary comparison of normalized T1 and non-contrast perfusion MRI assessments of regional lung disease in cystic fibrosis patients. J Cyst Fibros 16(2):283-290

15. Miller CA, Naish JH, Bishop P, Coutts G, Clark D, Zhao S et al (2013) Comprehensive validation of cardiovascular magnetic resonance techniques for the assessment of myocardial extracellular volume. Circ Cardiovasc Imaging 6(3):373-383

16. Messroghli DR, Moon JC, Ferreira VM, Grosse-Wortmann L, He T, Kellman P et al (2017) Clinical recommendations for cardiovascular magnetic resonance mapping of $\mathrm{T} 1, \mathrm{~T} 2, \mathrm{~T} 2 *$ and extracellular volume: a consensus statement by the society for cardiovascular magnetic resonance (SCMR) endorsed by the european association for cardiovascular imaging (EACVI). J Cardiovasc Magn Reson 19(1):75

17. Avants BB, Epstein CL, Grossman M, Gee JC (2008) Symmetric diffeomorphic image registration with cross-correlation: evaluating automated labeling of elderly and neurodegenerative brain. Med Image Anal 12(1):26-41

18. Naish JH, Kershaw LE, Buckley DL, Jackson A, Waterton JC, Parker GJM (2009) Modeling of contrast agent kinetics in the lung using T1-weighted dynamic contrast-enhanced MRI. Magn Reson Med 61(6):1507-1514

19. Hueper K, Parikh MA, Prince MR, Schoenfeld C, Liu C, Bluemke DA et al (2013) Quantitative and semiquantitative measures of regional pulmonary microvascular perfusion by magnetic resonance imaging and their relationships to global lung perfusion and lung diffusing capacity: the multiethnic study of atherosclerosis chronic obstructive pulmonary disease study. Invest Radiol 48(4):223-230

20. Carter P, Lagan J, Fortune C, Bhatt DL, Vestbo J, Niven R et al (2019) Association of cardiovascular disease with respiratory disease. J Am Coll Cardiol 73(17):2166-2177

21. Moosig F, Bremer JP, Hellmich B, Holle JU, Holl-Ulrich K, Laudien M et al (2013) A vasculitis centre based management strategy leads to improved outcome in eosinophilic granulomatosis and polyangiitis (Churg-Strauss, EGPA): monocentric experiences in 150 patients. Ann Rheum Dis 72(6):1011-1017

22. Schelbert EB, Sabbah HN, Butler J, Gheorghiade M (2017) Employing extracellular volume cardiovascular magnetic resonance measures of myocardial fibrosis to foster novel therapeutics. Circ Cardiovasc Imaging 10(6):i1

23. Lurz JA, Luecke C, Lang D, Besler C, Rommel K-P, Klingel $\mathrm{K}$ et al (2018) CMR-derived extracellular volume fraction as a marker for myocardial fibrosis: the importance of coexisting myocardial inflammation. JACC Cardiovasc Imaging 11(1):38-45

24. Gulati A, Jabbour A, Ismail TF, Guha K, Khwaja J, Raza S et al (2013) Association of fibrosis with mortality and sudden cardiac death in patients with nonischemic dilated cardiomyopathy. JAMA 309(9):896-908

25. Moravsky G, Ofek E, Rakowski H, Butany J, Williams L, RalphEdwards A et al (2013) Myocardial fibrosis in hypertrophic cardiomyopathy: accurate reflection of histopathological findings by CMR. JACC Cardiovasc Imaging 6(5):587-596

26. Higgins CB, Herfkens R, Lipton MJ, Sievers R, Sheldon P, Kaufman $L$ et al (1983) Nuclear magnetic resonance imaging of acute myocardial infarction in dogs: alterations in magnetic relaxation times. Am J Cardiol 52(1):184-188

27. Leach MO, Brindle KM, Evelhoch JL, Griffiths JR, Horsman MR, Jackson A et al (2005) The assessment of antiangiogenic and antivascular therapies in early-stage clinical trials using magnetic resonance imaging: issues and recommendations. Br J Cancer 92(9):1599-1610

28. Szczeklik W, Miszalski-Jamka T, Mastalerz L, Sokolowska B, Dropinski J, Banys R et al (2011) Multimodality assessment of cardiac involvement in churg-strauss syndrome patients in clinical remission. Circ J 75(3):649-655

29. Marmursztejn J, Guillevin L, Trebossen R, Cohen P, Guilpain P, Pagnoux C et al (2013) Churg-strauss syndrome cardiac involvement evaluated by cardiac magnetic resonance imaging and positron-emission tomography: a prospective study on 20 patients. Rheumatol 52(4):642-650

30. Miszalski-Jamka T, Szczeklik W, Sokołowska B, Karwat K, Belzak K, Mazur W et al (2013) Standard and feature tracking magnetic resonance evidence of myocardial involvement in churgstrauss syndrome and granulomatosis with polyangiitis (Wegener's) in patients with normal electrocardiograms and transthoracic echocardiography. Int J Cardiovasc Imaging 29(4):843-853

31. Yune S, Choi DC, Lee BJ, Lee JY, Jeon ES, Kim SM et al (2016) Detecting cardiac involvement with magnetic resonance in patients with active eosinophilic granulomatosis with polyangiitis. Int J Cardiovasc Imaging 32(Suppl 1):155-162

32. Wong TC, Piehler KM, Kang IA, Kadakkal A, Kellman P, Schwartzman DS et al (2014) Myocardial extracellular volume fraction quantified by cardiovascular magnetic resonance is increased in diabetes and associated with mortality and incident heart failure admission. Eur Heart J 35(10):657-664

33. Schelbert EB, Fridman Y, Wong TC, Abu Daya H, Piehler KM, Kadakkal A et al (2017) Temporal relation between myocardial fibrosis and heart failure with preserved ejection fraction: association with baseline disease severity and subsequent outcome. JAMA Cardiol 2(9):995-1006

34. Ricciardi MJ, Wu E, Davidson CJ, Choi KM, Klocke FJ, Bonow RO et al (2001) Visualization of discrete microinfarction after percutaneous coronary intervention associated with mild creatine kinase-MB elevation. Circulation 103(23):2780-2783

35. Schelbert EB, Cao JJ, Sigurdsson S, Aspelund T, Kellman P, Aletras AH et al (2012) Prevalence and prognosis of unrecognized myocardial infarction determined by cardiac magnetic resonance in older adults. JAMA 308(9):890-896

36. Cutillo AG, Chan PH, Ailion DC, Watanabe S, Rao NV, Hansen $\mathrm{CB}$ et al (2002) Characterization of bleomycin lung injury by nuclear magnetic resonance: correlation between NMR relaxation times and lung water and collagen content. Magn Reson Med 47(2):246-256

37. Claesson-Welsh L (2015) Vascular permeability-the essentials. Ups J Med Sci 120(3):135-143

38. Alamidi DF, Kindvall SSI, Hubbard Cristinacce PL, McGrath DM, Young SS, Naish JH et al (2016) T1 relaxation time in lungs of asymptomatic smokers. PLoS ONE 11(3):e0149760

39. Alamidi DF, Morgan AR, Hubbard Cristinacce PL, Nordenmark LH, Hockings PD, Lagerstrand KM et al (2016) COPD patients have short lung magnetic resonance $\mathrm{T} 1$ relaxation time. COPD 13(2):153-159

40. Stadler A, Jakob PM, Griswold M, Stiebellehner L, Barth M, Bankier AA (2008) T1 mapping of the entire lung parenchyma: influence of respiratory phase and correlation to lung function 
test results in patients with diffuse lung disease. Magn Reson Med 59(1):96-101

41. Stadler A, Jakob PM, Griswold M, Barth M, Bankier AA (2005) T1 mapping of the entire lung parenchyma: influence of the respiratory phase in healthy individuals. J Magn Reson Imaging 21(6):759-764

42. Cameron IL, Ord VA, Fullerton GD (1984) Characterization of proton NMR relaxation times in normal and pathological tissues by correlation with other tissue parameters. Magn Reson Imaging 2(2):97-106

43. Hartley RA, Barker BL, Newby C, Pakkal M, Baldi S, Kajekar $R$ et al (2016) Relationship between lung function and quantitative computed tomographic parameters of airway remodeling, air trapping, and emphysema in patients with asthma and chronic obstructive pulmonary disease: a single-center study. J Allergy Clin Immunol 137(5):1413-22.e12

44. Dunnil MS (1960) The pathology of asthma, with special reference to changes in the bronchial mucosa. J Clin Pathol 13(1):27-33

45. Baum C, Ojeda FM, Wild PS, Rzayeva N, Zeller T, Sinning CR et al (2016) Subclinical impairment of lung function is related to mild cardiac dysfunction and manifest heart failure in the general population. Int J Cardiol 218:298-304

46. Lam CSP, Lyass A, Kraigher-Krainer E, Massaro JM, Lee DS, Ho JE et al (2011) Cardiac dysfunction and noncardiac dysfunction as precursors of heart failure with reduced and preserved ejection fraction in the community. Circulation 124(1):24-30

47. Wannamethee SG, Shaper AG, Papacosta O, Lennon L, Welsh P, Whincup PH (2016) Lung function and airway obstruction: associations with circulating markers of cardiac function and incident heart failure in older men-the British regional heart study. Thorax 71:526-534
48. Agarwal SK, Heiss G, Barr RG, Chang PP, Loehr LR, Chambless LE et al (2012) Airflow obstruction, lung function, and risk of incident heart failure: the atherosclerosis risk in communities (ARIC) study. Eur J Heart Fail 14:414-422

49. Engström G, Melander O, Hedblad B (2010) Population-based study of lung function and incidence of heart failure hospitalisations. Thorax 65(7):633-638

50. Vasan RS, Sullivan LM, Roubenoff R, Dinarello CA, Harris T, Benjamin EJ et al (2003) Inflammatory markers and risk of heart failure in elderly subjects without prior myocardial infarction: the framingham heart study. Circulation 107(11):1486-1491

51. Kalogeropoulos A, Georgiopoulou V, Psaty BM, Rodondi N, Smith AL, Harrison DG et al (2010) Inflammatory markers and incident heart failure risk in older adults: the health $\mathrm{ABC}$ (health, aging, and body composition) study. J Am Coll Cardiol 55(19):2129-2137

52. Neukamm AM, Hoiseth AD, Hagve TA, Soyseth V, Omland T (2013) High-sensitivity cardiac troponin T levels are increased in stable COPD. Heart 99(6):382-387

53. Paulus WJ, Tschope C (2013) A novel paradigm for heart failure with preserved ejection fraction: comorbidities drive myocardial dysfunction and remodeling through coronary microvascular endothelial inflammation. J Am Coll Cardiol 62(4):263-271

Publisher's Note Springer Nature remains neutral with regard to jurisdictional claims in published maps and institutional affiliations. 